Hypothesis

\title{
Action potential propagation: ion current or intramembrane electric field?
}

\author{
Albert Martí1 ${ }^{1}$ Juan J. Pérez ${ }^{2}$ and Jordi Madrenas ${ }^{1}$ \\ ${ }^{1}$ Department of Electronics Engineering, Universitat Politècnica de Catalunya, Campus Nord, Building C4. C. Jordi Girona, \\ 1-3, 08034 Barcelona, Catalunya, Spain \\ ${ }^{2}$ Department of Chemical Engineering, Universitat Politècnica de Catalunya ETSEIB, Av. Diagonal 647, 08028 Barcelona, \\ Catalunya, Spain
}

\begin{abstract}
The established action potential propagation mechanisms do not satisfactorily explain propagation on myelinated axons given the current knowledge of biological channels and membranes. The flow across ion channels presents two possible effects: the electric potential variations across the lipid bilayers (action potential) and the propagation of an electric field through the membrane inner part. The proposed mechanism is based on intra-membrane electric field propagation, this propagation can explain the action potential saltatory propagation and its constant delay independent of distance between Ranvier nodes in myelinated axons.
\end{abstract}

Key words: Saltatory action potential propagation - Ion channels - Intra-membrane electric fields - Myelinated axon - Neuron membrane

\section{Introduction}

Nerve impulse propagation is a key element for understanding neural operation. In order to explain its propagation, several studies, works and models have been developed in the past. The work by Hermann (1879) devoted to model neuronal electrotonic potentials preceded the cable theory that was used a few years later and became a widely accepted model to explain action potential propagation in neuron axons. Cable theory has its origins in the mathematical developments made by Thomson (1855) aimed to explain the existing attenuation in under-water telegraph transmissions. In the 1940s Cole and Curtis (1941), Goldman (1943), Hodgkin and Katz (1949) adapted the cable theory to describe the conduction of nerve fibers. Years later, Hodgkin and Huxley (1952d) developed a set of equations to explain the results obtained in the study of ion traffic inside ion channels on the squid giant axon. These equations and their subsequent revisions (FitzHugh 1961, 1962; Nagumo et al. 1962; Morris and Lecar 1981; Hindmarsh and Rose 1984; Kistler et

Correspondence to: Albert Martí, Department of Electronics Engineering, Universitat Politècnica de Catalunya, Campus Nord, Building C4. C. Jordi Girona, 1-3, 08034 Barcelona, Catalunya, Spain E-mail: albert.marti.edo@gmail.com al. 1997; Loew 2012; Moore 2015) provided a mechanism that explains the generation of electric pulses in neurons. An important conclusion of these works was that the action potential is originated from the imbalance generated by ion channels traffic. Thus, currently accepted models are based on the propagation of electric pulses initiated at the ion channels that are conducted along the axon according to the cable theory. Rall (1962) complemented the cable theory for dendrite propagation by proposing the compartmental modeling approach, to explain the membrane active properties. Years later, Jack et al. (1975) developed the nonlinear cable theory providing a systematic and explanatory view of cable theory. Other authors conducted studies on cable theory into dendritic areas and as well on existing electric fields in the extracellular fluid (Nunez and Srinivasan 2006).

A few years ago Stühmer et al. (1989) and later Terlau and Stühmer (1998) performed a series of studies on the activation mechanism of sodium ion channels, proposing a model for channel structure and its operating mechanism. A few years later, Doyle et al. (1998) provided new insights into the working mechanism of potassium channels from the analysis of the crystallographic structure for the KcsA potassium channel solved at $3.2 \AA$ resolution. These studies suggested that potassium channels exhibit an arrangement of S4 segments, not aligned to the ion channel, that enable 
the movement of other S5-S6 segments, making possible channel opening and closing.

Recent work on voltage sensors (Bezanilla 2000), on the structure of the voltage-gated channels (Catterall 2000, 2010, 2012; Payandeh et al. (2011), on the displacement of the S4 segment (Horn 2004, 2005), on structural mechanism of the voltage-dependent gating ( $\mathrm{Li}$ et al. 2014) and on channels and pumps (Armstrong 2005) extended our knowledge about the structure and operating mechanism of ion channels, suggesting that the $S 4$ segment is key for activating the mechanism of channel opening.

In the present work, the hypothesis of action potential propagation by means of intramembrane electric fields is proposed. Various mechanisms for propagation in myelinated axons are reviewed, as well as the physical principles underlying this propagation. The proposed electric field propagation mechanism is described with its components and interactions. Each component of the mechanism is analyzed together with its structures and functions. The propagation requirements and energetic costs are analyzed.

\section{Action potential propagation mechanism in neurons}

Diverse models have been proposed in the past to explain action potential propagation in myelinated axons including the ion diffusion/ion repulsion (Islam 2004; Purves et al. 2004); ion drift (Pods et al. 2013) and proton hopping (Szasz et al. 2011; Kier and Tombes 2013). However, action potential propagation in myelinated axons exhibits effects that are not fully explained by these models. Thus, for example current models do not provide the reason for the absence of an action potential in the myelinated areas and their saltatory propagation. Another shortcoming of current models regards the action potential propagation time after present understanding of ion channel operation. Propagation time should include in addition to the time involved in travelling along the axon, the required time for the ion channels to open, involving the displacement of the channel segments plus the elapsed time to start ion circulation to generate the new action potential. Actually, current models do not consider the mechanical restructuring of the channel and its associated response time that can be the most time-consuming step (Ogden and Stanfield 1994). Finally, current models disregard important geometrical constrains of the myelinated axons. Thus, action potential propagation time between Ranvier nodes is constant regardless of the distance between them, being approximately $17 \mu$ s (Deutsch and Deutsch 1993). This result is consistent with the study reported by Waxman and Bennett (1972) where it was found a linear relationship between propagation velocity and axon diameters in myelinated axons with a slope $\approx 17 \mu$ s. Furthermore, there is a constant ratio between the myelinated zone diameter and the distance between Ranvier nodes of $\approx 100$ (Friede and Beuche 1985). Finally, myelinated axons have a ratio between the diameters of myelinated and unmyelinated areas, observed effect and validated mathematically first by (Rushton 1951) and subsequently (Chomiak and $\mathrm{Hu}$ 2009), with a value of $\mathrm{e}^{-1 / 2} \approx 0.6065$. Accordingly, the ratio of the distance between two Ranvier nodes and the axon diameter is 100 in the case of a myelinated axon zone and 164.88 is for the unmyelinated axon zone. These values are approximate and depend on the analyzed species and the degree of axon maturation.

In view of the points raised above let us analyze the feasibility of current model mechanisms. Ion diffusion/ion repulsion and ion drift model mechanisms are based on the displacement of sodium ions through the intracellular fluid between nodes. Ions generate the necessary potential difference on the unmyelinated zone of the membrane to open ion channels that permit inflow of new sodium ions.

These mechanisms do not explain the observed constant time propagation between two Ranvier nodes. In the case of the diffusion model mechanism, the time required for an ion to diffusion in a fluid can be estimated using the Einstein Smoluchowski equation:

$d=\sqrt{2 D t}$

Where $d$ is the mean square distance covered, $D$ is the diffusion coefficient and $t$ is time. The more general form to calculate the diffusion coefficient $D$ is by means of the Einstein-Nernst equation:

$D=\mu k T$

Where $\mu$ is the particle mobility, $k$ is the Boltzmann constant and $T$ is the absolute temperature and for electrically charged particles, there is a special form named electrical mobility equation:

$$
D=\frac{\mu_{q} k T}{Z_{i} e}
$$

Where $\mu_{q}$ is the electrical mobility of the charged particles and $Z_{i} e$ is the ion electric charge. Consequently, the diffusion coefficient used for a sodium ion contains the diffusion and repulsion effects, since it is calculated with the electric mobility equation that includes the electric mobility factor. Taking into account that sodium ions have a diffusion coefficient of $D=1.33 \times 10^{-9} \mathrm{~m}^{2} / \mathrm{s}$ (Atkins and de Paula 2006), during a time of $17 \mu$ s, ions will travel about $213 \mathrm{~nm}$. This distance is two orders of magnitude lower than the $20 \mu \mathrm{m}$ that corresponds to the minimal distance between Ranvier nodes for axons of $0.2 \mu \mathrm{m}$ diameter.

The diffusion mechanism has a three-dimensional behavior, while propagation is a one-dimensional, therefore 
the number of ions necessary for an increase in diameter grows cubically while the contribution grows linearly. This implies that the diffusion mechanism will produce a quadratic delay increase with distance among nodes while, as previously indicated, experiments show that it approximately remains constant. These facts clearly discard the diffusion mechanism.

In the case of the ion drift, propagation is based on the potential difference generated between ions that have crossed a specific sodium channels at a given Ranvier node and the resting potential of the next one. However, this model is not supported by the Debye and Huckel theory (1923). We have to consider that the transferred ions to intracellular fluid need to be hydrated and their electrical potential $V$ can be calculated using Debye and Huckel equation that incorporates an exponential attenuation factor:

$V=\frac{z q}{4 \pi \varepsilon_{0} \varepsilon_{r} r} e^{(-\kappa r)}$

where $z$ is the ion charge, $\varepsilon_{0}$ is the dielectric constant (8.8541 $\left.\times 10^{-12} \mathrm{~F} / \mathrm{m}\right), \varepsilon_{r} \approx 75$ is the relative dielectric constant of the cellular fluids (Lide 2005), $r$ is the distance and $\kappa$ is the Debye parameter, given by:

$\kappa=\left(\frac{2 F^{2} I}{\varepsilon_{0} \varepsilon_{r} R T}\right)^{1 / 2}$

Where $\mathrm{R}$ is the gas universal constant $(8.314 \mathrm{~J} / \mathrm{K} \mathrm{mol})$, $\mathrm{F}$ is the Faraday constant $(96485 \mathrm{C} / \mathrm{mol}), \mathrm{T}$ is the biological temperature $(310 \mathrm{~K})$, and $I$ is the ionic strength defined as:

$$
I=\frac{1}{2} \sum c_{i} Z_{i}^{2}
$$

Where $c_{i}$ are the concentrations of each of the various ion species present in the fluid and $Z_{i}$ is the charge of each ion. In order to compute $\kappa$, it is necessary to have an estimate of the ionic strength the intracellular fluid. Table 1 shows typical concentrations of ions present in the intracellular fluid.

According to Table 1, for the extracellular fluid, $I=$ $134.3 \mathrm{~mol} / \mathrm{m}^{3}$ and the corresponding $\kappa=1.208 \times 10^{9} \mathrm{~m}^{-1}$ and for the intracellular fluid, $I=143.0 \mathrm{~mol} / \mathrm{m}^{3}$ and $\kappa=1.247$ $\times 10^{9} \mathrm{~m}^{-1}$, respectively. As can be seen, the large values of $\kappa$ will produce a significant attenuation of the electric field for nanometric distances in both fluids.

Accordingly, the electric field produce by the sodium ions will vanish at distances longer than $100 \mathrm{~nm}$ due to the exponential decay. Consequently, hydrated ions cannot generate an electric potential strong enough to interact with ions located at the next Ranvier node.

Finally, the proton hopping mechanism, also called Grotthuss mechanism (Szasz et al. 2011) is a quantummechanical process based on the recombination-dissociation process of hydronium ions. According to the authors by this
Table 1. Example of ion concentration in a neuron

\begin{tabular}{lcc}
\hline \multirow{2}{*}{ Ion } & \multicolumn{2}{c}{ Concentration $(\mathrm{mEq} / \mathrm{l})$} \\
\cline { 2 - 3 } & interstitial & intracellular \\
\hline $\mathrm{Na}^{+}$ & 139.0 & 14.0 \\
$\mathrm{~K}^{+}$ & 4.0 & 140.0 \\
$\mathrm{Ca}^{2+}$ & 1.2 & 0.0 \\
$\mathrm{Mg}^{2+}$ & 0.7 & 20.0 \\
$\mathrm{Cl}^{-}$ & 108.0 & 4.0 \\
$\mathrm{HCO}_{3}{ }^{-2}$ & 2.0 & 11.0 \\
$\mathrm{HPO}_{4}{ }^{-2}, \mathrm{H}_{2} \mathrm{PO}_{4}{ }^{-2}$ & 0.5 & 1.0 \\
\hline
\end{tabular}

(Hall and Guyton 2011).

mechanism protons propagate approximately ten times faster than in ion diffusion. Accordingly, the expected diffusion coefficient will be $93.1 \times 10^{-9} \mathrm{~m}^{2} / \mathrm{s}$, ten times the actual diffusion coefficient (Atkins and de Paula 2006). However, using this mechanism, protons will only travel $1.78 \mu \mathrm{m}$ in $17 \mu \mathrm{s}$, still far from the $20 \mu \mathrm{m}$ that corresponds to the minimal distance between Ranvier nodes for axons of $0.2 \mu \mathrm{m}$ diameter. This leads to conclude that ion channel opening due to proton accumulation considering protons travelling between two consecutive Ranvier nodes is not a valid mechanism.

\section{The electric field propagation mechanism}

We propose in the present work an action potential propagation mechanism based on the electric field produced by ions when crossing the ion channel. This field will be transmitted through the low-permittivity inner part of the membrane.

Electric field models have been used in the past to explain intercellular propagation in myocardial excitable cells (Ruffner et al. 1980; Sperelakis 2002). Actually, we propose in the present work to extend these ideas to the membrane inner part to explain the mechanism of action potentials. Similar ideas were used in a recent work devoted to the physiology of neural nanocompartments, where the authors proposed an extension of the Cable theory and the Goldman-Hodgkin-Huxley-Katz models based on the Poisson-Nernst-Planck approximation and electrodiffusion to obtain accurate results (Holcman and Yuste 2015).

In order to introduce the electric field propagation mechanism, let us first consider the axon segment between two Ranvier nodes as an electric field communication system, composed by a transmitter, a communication channel, and a receiver.

The sodium voltage-gated channels present in axons operate as a transmitter. They are primarily responsible for the generation and propagation of action potentials along myelinated and unmyelinated axons. In the myelinated 
axons, action potentials cannot be measured continuously along the axon. Instead, they can only be measured on unmyelinated areas, i.e. at the Ranvier nodes and at the initial axon segment. This disability to detect the action potential is due to the low presence of ion channels in the myelinated zones (less than 4\%) (Shrager 1987, 1988, 1989).

The low-permittivity of the axonal membrane inner zone operates as the transmission system communication channel. The receiver function is performed by sodium channels located at the next Ranvier nodes. In fact, those channels operate as transmitters for the next Ranvier node, so ion channels can be considered as transceivers.

When the electric potential difference change exceeds a certain threshold, voltage-activated sodium channels open with the subsequent flow of sodium ions through the channels and an action potential is produced, presenting the dynamics described by Hodgkin and Huxley (1952a, 1952b, 1952c).

A fundamental effect produced when ion channels turn on is the emergence, in the channel, of an electric field generated by the ions crossing the channel, and therefore the propagation of this electric field inside the membrane on the plane perpendicular to the channel and longitudinal to the membrane, as depicted in Fig. 1.

The assumption of the proposed mechanism is that the electric field inside the hydrophobic part of the membrane during the action potential can open nearby ion channels by means of electric field interaction. In unmyelinated axons, the ion channel scattering generates low levels of electric field. In unmyelinated areas of myelinated axons where the ion channel concentration is very high and channel opening occurs synchronously, stronger electric field is generated. Low-level electric fields allow nearby propagations "short jumps" (Neishabouri and Faisal 2014), while high level ones permit both nearby and distant propagation "saltatory propagation" (Huxley and Stämpfli 1949).

Instead of the classic explanation of charge displacement, the proposed action potential propagation mechanism is based on the electric field generated by dehydrated ions in their traffic through the sodium channels and its propagation inside the cell membrane, which operates as a coaxial guide. The ion traffic of the potassium channels and the operation of sodium-potassium pump are needed to restore initial conditions to restart the process.

\section{Mechanism components}

In the generation, propagation and regeneration of the action potential, two main elements are involved: the cell membrane and the ion channels.

\section{Cell membrane}

The cell membrane is a lipid bilayer of approximately $4 \mathrm{~nm}$ thickness (Milo and Philipps 2015), mainly composed of proteins, polar phospholipids and glycolipids and, in a much smaller amount, of sphingolipids and cholesterol.

The composition of the two membrane layers is asymmetric, because they do not contain the same type of phospholipids. The outer layer is composed by uncharged and zwitterionic phospholipids with null net electric charge or electrically neutral. The more abundant phospholipid is phosphatidylcholine, which presents two electric charges on its neutral polar head, a positive one outside the cell membrane and a negative one inside it. The inner layer is composed of uncharged phospholipids, null net electric charge phospholipids and negative electric charge phospholipids. The more abundant phospholipid is phosphatidylethanolamine, with two electric charges on its neutral polar head, being the positive charge outside of the cell membrane. The two phospholipid types with electric negative charge are phosphatidylserine and phosphatidylinositol. They can only be found in this membrane layer, and in small quantities. Cholesterol can be found in both membrane layers.

This membrane composition allows the existence of two clearly delimited areas. First area, the outermost is mainly composed of polar heads. A second zone which occupies the central part is composed of the hydrophobic tails. The outer zone has a permittivity approximately equal to 50 (Hobbie and Roth 2015) and a thickness about $0.5 \mathrm{~nm}$. The internal area has a permittivity approximately equal to 2.2 (Simeonova and Gimsa 2006; Gramse et al. 2013; Hobbie and

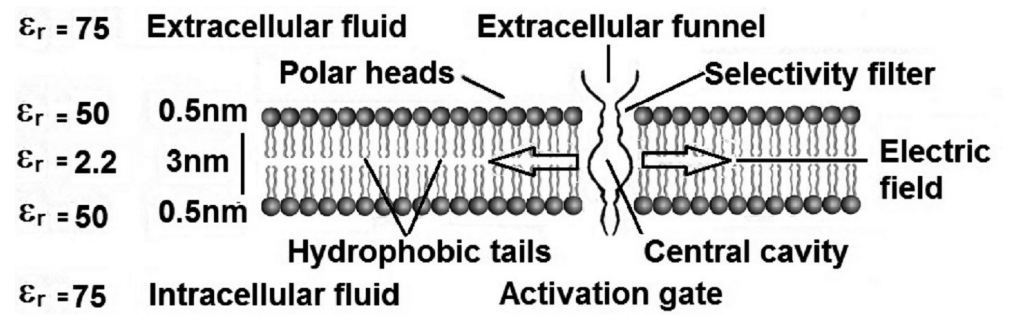

Figure 1. Membrane composed of phospholipids with two clearly defined zones: polar heads and hydrophobic tails. The arrangement of the polar heads allows the creation of a central communication channel with low permittivity $(\varepsilon r)$ and two equipotential surfaces that confine and protect the propagation of electric fields generated by ion channels. In the center a sodium channel sketch with the different parts of structure is shown: Extracellular

funnel, selectivity filter, central cavity and activation gate. The electric field generated by the ion accumulation in central cavity can be propagated by the low permittivity zone. 
Roth 2015), and a thickness about $3 \mathrm{~nm}$. A simple membrane model is depicted in Figure 1.

The axon, with its circular section and the double lipid layer, exhibits coaxial cylinder structure. The external and internal cylinder walls are composed of polar heads. This phospholipid structure presents a certain degree of mobility. Together with the presence of hydrated ions in the cell fluids, pseudo-equipotential surfaces are created, that prevent interference, allowing the axon membrane to safely propagate the electric fields.

The structure of the membrane and its special features in the myelinated axon provide us with very important information on the mechanism of propagation by electric fields.

\section{Myelinated axon geometry and electric field propagation}

The general solution of the Poisson equation for the electrostatic potential $\psi(\mathrm{r})$ is given by:

$$
\psi(\vec{r})=\frac{1}{4 \pi \varepsilon} \int \frac{\rho\left(r^{\prime}\right) d V\left(r^{\prime}\right)}{|\vec{r}-\vec{r}|}
$$

where $\rho\left(\mathrm{r}^{\prime}\right)$ is the charge distribution in volume, and $\left|\vec{r}-\vec{r}^{\prime}\right|$ is the distance between the charge differential element and the point where potential is calculated.

Considering a simplified charged ring with linear charge distribution $\lambda$ (Fig. 2), the particular solution of Eq. 7 becomes:

$$
\psi(\vec{r})=\frac{1}{4 \pi \varepsilon} \int_{0}^{l} \frac{\lambda d l}{|\vec{r}-\vec{r} \prime|}
$$

where $d l$ is the line differential along the ring. Applying the properties of the myelinated axon:

$$
d=2 x \approx \frac{z}{164.88}
$$

where $d$ is the Ranvier node diameter. Clearly $x<<z$ so we can approximate:

$\left|\bar{r}-\bar{r}^{\prime}\right| \cong Z$

Assuming constant ion channel distribution and thus constant linear charge density in Ranvier node, combining Eq. 8, 9 and 10, we obtain the Coulomb potential:

$$
\psi(z)=\frac{1}{4 \pi \varepsilon} \int_{0}^{l} \frac{\lambda}{z} d l=\frac{\lambda}{4 \varepsilon \cdot 164.88}
$$

It can be concluded that if the internode length $z$ scales with the axon diameter $d$, the potential at distance $z$ is constant and depends only on the Ranvier node linear charge density and the membrane internal permittivity. This is a consequence of the proportionality between internode distance and axonal diameter. Any diameter variation,

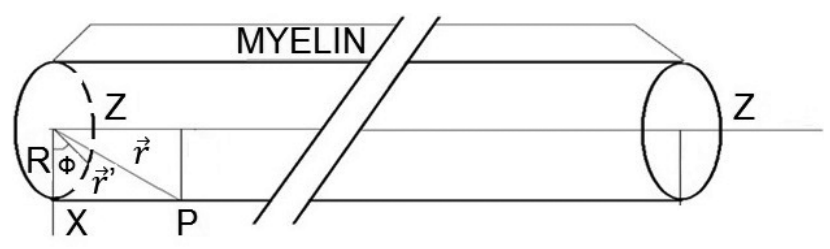

Figure 2. Ideal geometry sketch of the myelinated axon. R, axon radius in unmyelinated zone; $\mathrm{P}$, point of study for the electric potential; $\vec{r}^{\prime}$, vector that indicates the position of the differential charge element; $\vec{r}$, vector that indicates the position of the point $\mathrm{P} ; \Phi$, angle to integrate; $\mathrm{Z}$, propagation coordinate, $\mathrm{X}$, transversal coordinate.

assuming uniform channel density, implies an increase of the channel number and therefore an increase in the total number of ions crossing the channels. This increase of ions compensates for the longer distance among nodes, producing the same potential value at the destination node. Here also a single mechanism gives a reasonable explanation to the equidistance between nodes and proportionality with the axonal diameter. All electric charges crossing the membrane on a Ranvier node synchronously open all channels of the following node, regenerating the electric field in next node channels.

\section{Propagation of the electric field through an axon low-permittivity area}

The electric field propagates through the low-permittivity intra-membrane medium, at a speed close to the light in vacuum. Therefore, it can reach the next node of Ranvier in negligible time compared to other reviewed model mechanisms. As a consequence, the propagation time becomes only dependent on channel opening and ion trespassing mobility.

In regard to the electric field propagation, the low-permittivity region is limited by the polar head dipoles that guide and lead the field through the axon. Furthermore, despite the electric field in the center of the membrane is longitudinal, due to the polar head dipoles the electric field will be bent.

The electric field propagation model mechanism can also explain the propagation of action potentials in the unmyelinated axons. Ion channel density in unmyelinated axons is smaller than in myelinated ones. This generates in turn lower synchronous ion traffic and therefore, a lower electric field intensity, which translates into a shorter range to open ion channels. Propagation is also saltatory in small jumps, although it is observed as a continuous propagation of the action potential at the current measurement scale capability.

In both myelinated and unmyelinated axons, the electric field generated by ion traffic, besides opening the nearby channels, also collaborates in opening the distant channels. In myelinated axons this collaborative activation energy value corresponds to the series $(1,1 / 2,1 / 3,1 / 4, \ldots)$ although 


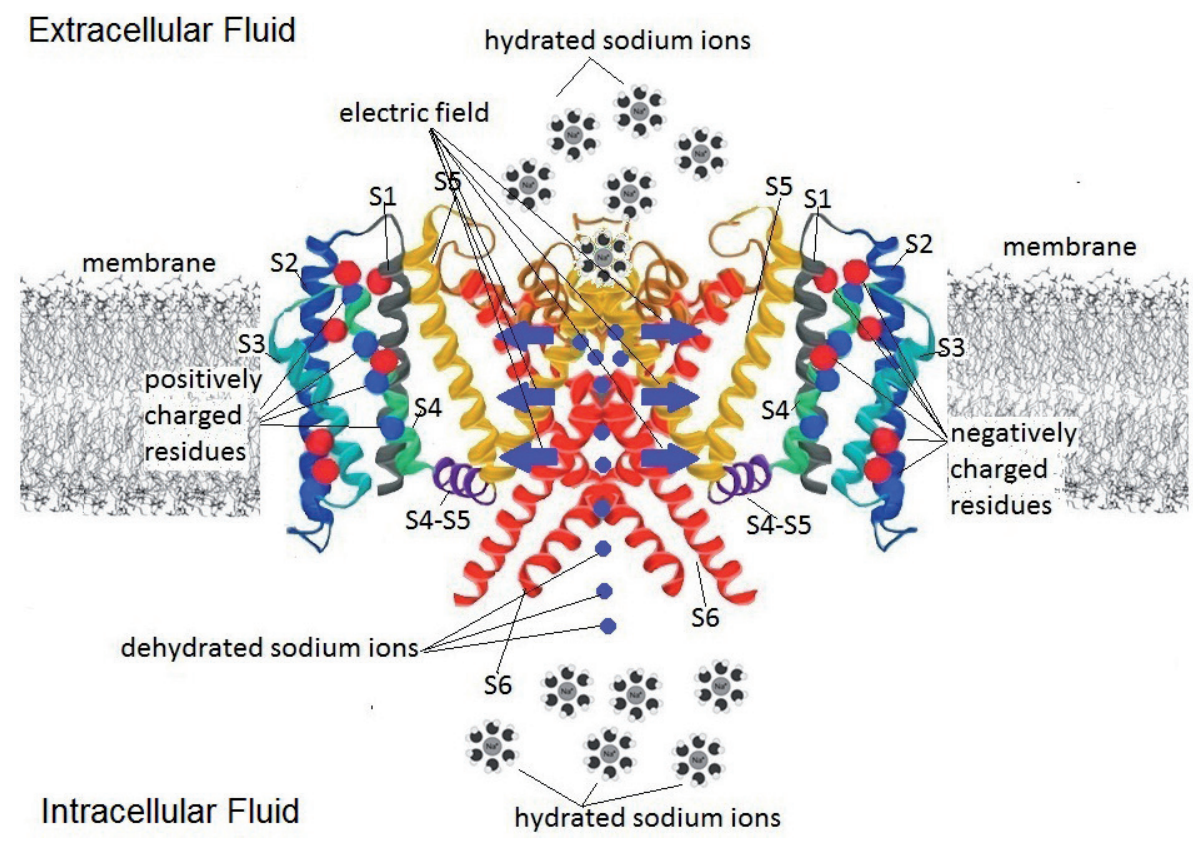

Figure 3. Crystallographic structure of the sodium channel with six segments and the membrane around, cellular fluids with their hydrated ions and electric field generated by the dehydrated ions inside the channel. Adapted from the crystallography of the sodium channel PDB ID: 3RW0 Crystal structure of the NavAb voltage-gated sodium channel (Met221Cys, 2.95 A). some additional factors can attenuate the collaborative effects, as for instance the higher permittivity level at Ranvier nodes due to the presence of ion channels.

\section{Ion channels, sensors and exciter}

Sodium and potassium channels are responsible for the different phases of the action potential. As shown in Fig. 3, the alignment of ion channel central cavity and the lowpermittivity cell membrane zone allows propagation of the electric field generated by ion traffic through channels.

Each type of ion channel presents a different electric activation potential, depending on it structure and composition, as shown in Table 2 .

The voltage-gated sodium channels have a shape of a $14 \mathrm{~nm}$ high truncated cone, with $12 \mathrm{~nm}$ diameter at its widest part and between 7 and $10 \mathrm{~nm}$ at its narrowest part (Sato et al. 1998). Crystallographic studies of sodium channels (Catterall 2014) describe them as a longitudinal

Table 2. Activation voltage of potassium $\mathrm{Kv}$ and sodium Nav ion channels

\begin{tabular}{lc}
\hline Channel & $\mathrm{mV}$ \\
\hline Kv1.1 & 27.0 \\
Kv1.2 & -35.0 \\
Kv1.4 & -34.0 \\
Kv3.1 & 16.0 \\
Nav1.6 & -37.7 \\
\hline
\end{tabular}

(Catterall et al. 2005; Gutman et al. 2005). structure with four distinct parts: extracellular funnel, selectivity filter, central cavity and activation gate, as depicted in Figure 1 and 3.

Sodium channels cyclically operate under tree states: open, closed and inactive. Open and closed states are function of the segment structural arrangement. The inactive state is a function of the activation gate. The state cycle starts from closed, open, inactive and turns again to closed state. The standard excitation method used to open ion channels is through the application of an electric potential difference between the two cell membrane layers.

\section{Description of channel elements}

The $\mathrm{S} 4$ segments are located in the channel structure in such a way that allow a movement towards the extracellular side with a certain angle relative to the channel axis by the extracellular funnel presence. The tilted position direction of S4 segment allow to trigger the displacement with a transversal electric field to the membrane or with a longitudinal electric field curved by membrane transversal electric field. Since the S4 segment is tilted the presence of a longitudinal intramembrane field alters the balance between the S4 segment charged residues and those charged residues in segments S1, S2 and S3 (Fasman 1990), moving the charged residues and the S4 segment to the extracellular side in a spiral motion through the protein structure (Stühmer et al. 1989; Horn 2004). The movement of positive electric charges causes the displacement of the four S4 segments. This displacement is transmitted through the existing link between segments S4-S5, inducing a flexion-torsion movement of the S5-S6 
segments in the four domains. The interrelationship between the S5-S6 segments of the four domains causes a synchronized movement that generates the channel opening (Catterall 2010, 2012).

The channel central cavity diameter is wide enough to allow the traffic of two or three dehydrated sodium ions simultaneously (Charabarti et al. 2013) which makes possible the presence of a substantial quantity of ions inside that cavity. The ion channel density and the ion concentration inside each channel are the factors that determine the maximum number of ions able to produce electric field inside the membrane. The point is whether the sum of electric fields generated by the ions travelling through the open channels can provide enough energy to activate voltage dependent channels, mainly sodium ones, located nearby or at substantial distances.

The low ion channel concentration in the unmyelinated axons permits opening only the ion channels located nearby. This effect can be associated with a continuous propagation effect of the action potential. In contrast, the high channel concentration in the Ranvier nodes allow the opening of ion channels located at substantially longer distances, as the electric field becomes much stronger due to the accumulation effect. A sketch of channel distribution at the Ranvier node is depicted in Figure 4A and 4B.

In mature myelinated axons, the most abundant ion channels are the Nav1.6 - principal initiator of the propa- gation - that are uniformly distributed in the Ranvier node zone and in the axon initial segment as depicted in Fig. 4A (Sheng and Wyszynski 1992; Caldwell et al. 2000; Boiko et al. 2001, 2003; Rush et al. 2005; Wittmack et al. 2005; Morris and Juranka 2007; Osorio et al. 2010; Camilla 2011; Chen and Chung 2012; Robbins and Tempel 2012).

\section{Propagation requirements}

According to current knowledge of ion channels, cell membrane and cellular fluids, the proposed propagation mechanism based on the electric field is capable to explain the specific properties and requirements of the action potential propagation in the myelinated axon.

\section{Constant-time propagation between consecutive Ranvier nodes}

The action potential total propagation delay between consecutive Ranvier nodes has three components: the signal propagation time between nodes, the sodium channel opening mechanism response time and, the sodium ion transport time to transfer into the channel the required amount of sodium ions that allow the generation of the necessary electric field to open the channels of the next Ranvier node.

\section{A}

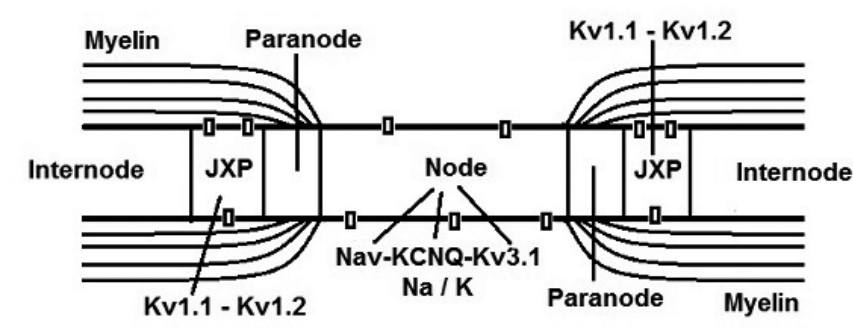

B

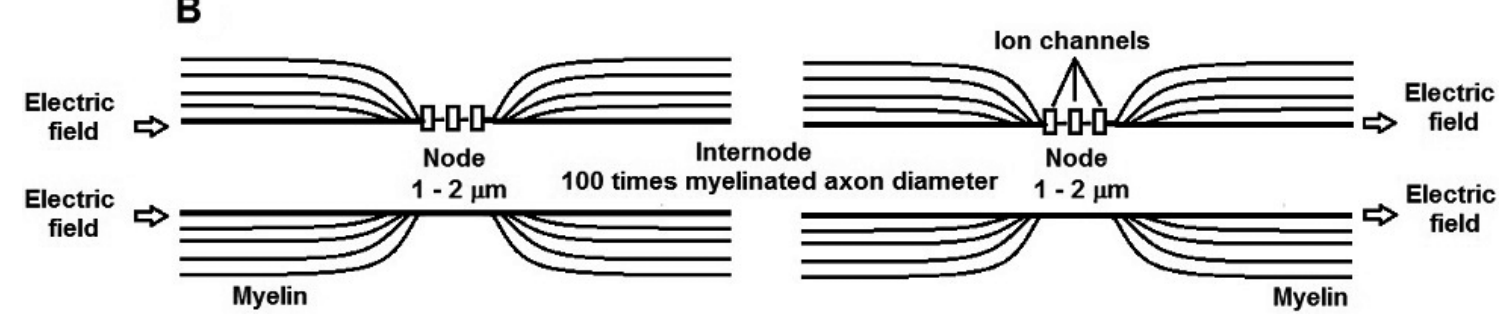

Figure 4.A. Ion channel distribution in a Ranvier node. In the internode zone, the concentration of ion channels is extremely low, in the juxtaparanode zone (JXP in figure) Kv1.1 and Kv1.2 channels are found. The paranode zone is the myelin junction zone, with virtual absence of ion channels. Finally, in the node zone, Nav1.6, Nav1.2, Kv3.1 and KCNQ (delayed rectifier) channels are found, in a very high concentration. This very high concentration of channels and the ions traffic inside the channels generate an electric field with sufficient intensity to open next channels. B. Description of electric field propagation between two Ranvier nodes. There we can see the dimensions of the Ranvier nodes and the proportions existing between the myelinated diameter of the nodes and the distance between nodes, as well as the propagation of the electric field through inner low permittivity zone of the membrane. 
As indicated above, the propagation speed of an electric field in media with low permittivity coefficients, as the nonpolar zone of the hydrophobic tails $\left(\varepsilon_{\mathrm{r}}=2.2\right)$, is high close to the light speed in vacuum. The distances between Ranvier nodes give sub-picosecond propagation times. Thus, this time can be neglected in front of the total propagation delay $(17 \mu \mathrm{s}$.$) . Channel opening time involves the coordinated$ movement of the four S4 segments and the spatial relocation of the channel four groups of S5-S6 segment generators. The time invested for this molecular motion with a patch clamp excitation (Ogden and Stanfield 1994) is about 10-20 $\mu$ s. For a longitudinal electric field this value is unknown, although their value will be similar or even higher. The ion transit speed through the ion channels is in the order of $6 \times 10^{6}$ ions/s, which implies about $1 \mu$ s or $2 \mu$ s to fill the channel with ion stream and thus generate the needed electric field. Despite the uncertainty on the exact delay of ion transit and of channel opening, the fact that all of them are in the microsecond order, with the proposed mechanism it is logic assigning all $17 \mu$ s to the processes associated with ion channels, since the electric field propagation delay is negligible. This approach explains the action potential propagation characteristics in the myelinated axons in constant time, regardless of the distance between nodes, according to biological knowledge of the membranes and ion channels.

\section{Equidistance between nodes and proportionality with the axonal diameter}

The constant ratio in the myelinated axon between the myelinated and unmyelinated area diameter is related to the maintenance of the axon structure. High concentration of ion channels in the Ranvier nodes can generate a high presence of $\mathrm{Na}$ and $\mathrm{K}$ ions inside the membrane at the opening time and consequently, a high-intensity electric field. The membrane structure in the myelinated area is mainly composed by lipids, which are phospholipids with polar heads in a large part. This myelin layer accumulation in membrane limits the mobility of its polar phospholipids against high electric field. Besides structure consistency, the myelin layers have the function to prevent the possible generation of ion currents in the few existing channels in this zone, allowing the existence of order and coordination in the propagation of the action potential, preventing possible interferences generated by opening of channels located in myelinated areas.

The node equidistance is the result of maximizing the distance that can be covered by the action potential propagation mechanism. In the proposed electric field propagation hypothesis, it is the maximum distance at which the energy provided is sufficient to open the ion channels of the next Ranvier node.

\section{Ion channel traffic}

To determine an approximate number of charges required to generate an electric potential that can open sodium channels located at a distance of 164.88 times the Ranvier node diameter, we need to compute the electric energy $U$ required for the activation of a sodium channel, estimated in 11193.53 $\times 10^{-23} \mathrm{~J}(16.1 \mathrm{kcal} / \mathrm{mol})$ (Chowdhury and Chanda 2012). Using Eq. 11 the energy $U$ can be computed as:

$U=\psi(z) n q=\frac{\lambda n q}{4 \varepsilon_{0} \varepsilon_{r} 164.88}$

where $n$ is the number of electric charges in the S4 segments estimated between 16-28 (Catterall 2012), $q$ is the electron charge $\left(1.602 \times 10^{-19} \mathrm{C}\right)$, $\varepsilon_{0}$ is the vacuum permittivity $\left(8.854 \times 10^{-12} \mathrm{~F} / \mathrm{m}\right)$ and $\varepsilon_{r}=2.2$, is the medium relative dielectric constant.

The linear charge density can be expressed as:

$\lambda=I c \sigma N l q$

where $I c$ is the number of ions inside a channel, $\sigma$ is the density of channels $\sigma \approx 350$ channels $/ \mu \mathrm{m}^{2}$ ) (Debanne et al. 2011), and $\mathrm{Nl}$ is the Ranvier node length. We assume the smaller value for the worst case $N l \approx 1 \mu \mathrm{m}$ (Arancibia-Carcamo and Attwell 2014).

Replacing Eq. 13 in Eq. 12,

$U=\frac{n I c \sigma N l q^{2}}{4 \varepsilon_{0} \varepsilon_{r} 164.88}$

we obtain the number of ions inside a channel that are necessary to open the channels at the next Ranvier node:

$I c=\frac{659.52 U \varepsilon_{0} \varepsilon_{r}}{n \sigma N l q^{2}}$

Replacing with actual values, for 16 charges in S4 segments, $I c \approx 10$ ions. For 28 charges in $\mathrm{S} 4$ segments, $I c \approx 6$ ions. These are reasonable figures, close to the ion channel capacity. Also, higher channel density or longer node length would require fewer ions inside the channels. Furthermore, the collaborative effect to open the channels could easily reduce down to 4 the number of needed ions in the channels.

The channel, in the central cavity area aligned with the $3 \mathrm{~nm}$ hydrophobic tail zone, allows the travel of two or three sodium ions simultaneously, which makes the presence of more than 4 ions in the channel feasible.

Another magnitude interesting to compute is the potential created by the conducting ion channels at the next node as obtained in Eq. 11. It results $\psi(Z)=I c 4.36 \mathrm{mV}$. For 4 ions inside a channel $(I c=4), \psi(Z)=17.44 \mathrm{mV}$, which is above the typical $15 \mathrm{mV}$ above resting potential required to produce an action potential, i.e. to open the sodium channels. 


\section{Dehydration ions}

In the previous calculations it has been assumed that ions are dehydrated, otherwise the correction of the Debye-Hückel (Eq. 4), should be applied. A key aspect is verifying the capability and conditions under which sodium ions, subject to hydration in cellular fluids, become dehydrated and thus are able to propagate the electric field during their transit through the channels.

The hydration effect produces a significantly a greater radius of the system ion-dipole than the single ion, as shown in Table 3.

For the sodium ion, the radius grows about 3.5 times when hydrated. Its hydration energy is $-73063.71 \times 10^{-23} \mathrm{~J}(-440.0 \mathrm{~kJ} /$ $\mathrm{mol})$. In the sodium channel, the selection filter has a square structure with a side width of $460 \mathrm{pm}$, presenting in each corner a Glu177 residue with a total of four negative charges, allowing only the transit of positively charged ions. The 460 pm channel width allows the transit of all dehydrated ions present in the extracellular fluid and in the intracellular fluid (Catterall 2012). The closely placed positive ions present in the extracellular funnel at the time of the channel opening are attracted by the four negative charges present in the selection filter. This interaction between the hydrated ion and the four negative charges increases the kinetic energy of the ion-dipole system. This energy increase would allow a complete or almost complete dehydration of the ion.

Using the Debye-Hückel model Eq. 4, the energy equation becomes:

$U=\frac{z q^{2}}{4 \pi \varepsilon_{0} \varepsilon_{r} r} e^{(-\kappa r)}$

Applying the sodium hydration energy and $\kappa$ values in the extracellular funnel, we obtain $r \approx 16.5 \mathrm{pm}$ to the selectivity filter. The sodium ions pass at $223 \mathrm{pm}$ of the four negative charges so the electrostatic energy is not enough to completely dehydrate the ions and they enter partially dehydrated in the channel.

The ion entrance into the channel involves a change of medium (Qi and Sokabe 1999) and therefore the need to acquire enough energy (Born 1920), according to the equation of Born:

$U=\frac{q^{2}}{8 \pi \varepsilon_{0} a}\left(\frac{1}{\varepsilon_{r 2}}-\frac{1}{\varepsilon_{r 1}}\right)$

where $a=102.0 \mathrm{pm}$ is the sodium ion radius.

The ions travel from the extracellular medium with $\varepsilon_{r} \approx 75$ to the central cavity hydrophobic medium (Aguilella-Arzo et al. 2009) with $\varepsilon_{r} \approx 30$, increasing their energy in 2261.36 $\times 10^{-23} \mathrm{~J}$. The ions increased, in the partial dehydration process, their energy by $4507.93 \times 10^{-23} \mathrm{~J}$. This amount of energy is thus enough to allow the change of medium and the transit through the channel.
Table 3. Ion radii, hydration radii and hydration energy of the ions in the cell fluids

\begin{tabular}{lccc}
\hline Ion & $\begin{array}{c}\text { Ion radii } \\
(\mathrm{pm})\end{array}$ & $\begin{array}{c}\text { Hydration } \\
\text { radii }(\mathrm{pm})\end{array}$ & $\begin{array}{c}\text { Hydration energy } \\
(\mathrm{kJ} / \mathrm{mol})\end{array}$ \\
\hline $\mathrm{Na}^{+}$ & 102.0 & 358.0 & -440.0 \\
$\mathrm{~K}^{+}$ & 138.0 & 331.0 & -372.0 \\
$\mathrm{Ca}^{2+}$ & 100.0 & 412.0 & -1656.0 \\
$\mathrm{Mg}^{2+}$ & 72.0 & 428.0 & -2049.0 \\
$\mathrm{Cl}^{-}$ & 181.0 & 332.0 & -315.0 \\
\hline
\end{tabular}

(Conway 1981; Marcus 1991; Lide 2001)

The water molecules do not increase their energy level and cannot change of medium. As the sodium ions reach the necessary energy level they enter dehydrated into the channel.

\section{Energy costs}

The sodium channel opening process does not have any energy cost, because the energy required for that purpose is provided by the electric field produced by the ions in transit through channels. The sodium ion current from the extracellular fluid to the intracellular fluid presents neither energy cost because the required energy is provided by the imbalance of sodium ion concentration between the two fluids.

The subsequent opening of potassium channels does not have energy cost, because the energy required is provided by the electric field produced by the sodium ions in transit, and the current generated between the intracellular fluid and the extracellular fluid do not have energy cost either, since the energy is provided by the imbalance of potassium ion concentrations between the two fluids.

The only energy cost that the proposed mechanism presents is to restore the initial concentrations of sodium and potassium in the cellular fluids. The sodium-potassium pump is the responsible for this task using the energy provided by the adenosine triphosphate (ATP), and therefore the ATP contribution is the only form of energy necessary for the mechanism operation. This energy cost (Aiello and Bach-y-Rita 2000; Wang et al. 2015) corresponds to the ion transfer in the opposite direction to the concentrations. Since the proposed propagation mechanism is based on the ion accumulation inside the channel, the necessary ion transfer from one fluid to another can be minimal so that the energy cost for concentration restoration is also minimal.

\section{Conclusions}

The hypothesis proposed in the present work that action potential propagation mechanism is due to the electric field generated by ion trafficking through the channels and 
propagated inside the hydrophobic region of the neuron membrane, agrees well with current knowledge about the structure and function of cell membranes and ion channels.

The region between the two phospholipid layers forming the membrane, with the polar heads and ionic fluids as equipotential surface and the hydrophobic tails as low-permittivity communication channel, meets with the requirements to be a stable channel.

Ion channels are the elements responsible for detecting and regenerating the action potential, sensing, through the displacement of S4 segments and regenerating, through their capability to drive ions and to generate electric fields. This occurs when the level of electric potential exceeds the activation threshold and the flow of ions appears, generating a new electric field capable to open the surrounding ion channels of unmyelinated axons or in the next Ranvier node of myelinated axons.

Thus, presently proposed mechanism fulfills with the established premises and the propagation characteristics of both unmyelinated and myelinated axons. According to theoretical calculations, a few ions inside sodium channels suffice to open ion channels in the next Ranvier node. The collaborative effect application allows opening the channels with only 4 ions inside each channel. The constant-time propagation between Ranvier nodes agrees with the electric field mechanism because propagation time is independent to the distance, and only depends on the ion channel opening mechanism delay. The axonal diameter and the distance between Ranvier node constant ratio corresponds to the farthest point where a reasonable number of sodium ions can generate a strong enough electric field to open ion channels.

In summary, the proposed action potential propagation mechanism through the electric fields generated by ion trafficking through the channels meets the properties of action potential transmission in axons using a consistent approximation with the current knowledge.

\section{References}

Aguilella-Arzo M, Andrio A, Aguilella VM, Alcaraz A (2009): Dielectric saturation of water in a membrane protein channel. Phys. Chem. Chem. Phys. 11, 358-365 https://doi.org/10.1039/B812775A

Aiello GL, Bach-y-Rita P (2000): The cost of an action potential. J. Neurosci. Methods 103, 145-149 https://doi.org/10.1016/S0165-0270(00)00308-3

Arancibia-Carcamo L, Attwell D (2014): The node of Ranvier in CNS pathology. Acta Neuropathol. 128, 161-175 https://doi.org/10.1007/s00401-014-1305-Z

Armstrong CM (2005): Pumps, Transporters, and Ion Channels. Studies on Their Structure, Function, and Cell Biology. Kluwer Academic/Plenum Publishers, New York
Atkins PW, de Paula J (2006): Physical Chemistry. (8th ed.), pp. 775, WH Freeman and company, New York

Bezanilla F (2000): The voltage sensor in voltage-dependent ion channels. Physiol. Rev. 80, 555-592

Boiko T, Van Wart A, Caldwell JH, Levinson SR, Trimmer JS, Matthews G (2003): Functional specialization of the axon initial segment by isoform-specific sodium channel targeting. J. Neurosci. 23, 2306-2313

Boiko T, Rasband MN, Levinson SR, Caldwell JH, Mandel G, Trimmer JS, Matthews G (2001): Compact myelin dictates the differential targeting of two sodium channel isoforms in the same axon. Neuron 30, 91-104

https://doi.org/10.1016/S0896-6273(01)00265-3

Born MZ (1920): Volumen und hydratation-swarme der ionen. Z. Phys. 1, 45-48 https://doi.org/10.1007/BF01881023

Caldwell JH, Schaller KL, Lasher RS, Peles E, Levinson SR (2000): Sodium Nav1.6 is localized at nodes of Ranvier, dendrites, and synapses. PNAS 97, 5616-5620 https://doi.org/10.1073/pnas.090034797

Camilla SJ (2011): Neuronal trafficking of voltage-gated potassium channels. Mol. Cell. Neurosci. 48, 288-297 https://doi.org/10.1016/j.mcn.2011.05.007

Catterall WA (2000): From ionic currents to molecular mechanisms: the structure and function of voltage-gated sodium channels. Neuron 26, 13-25 https://doi.org/10.1016/S0896-6273(00)81133-2

Catterall WA (2010): Ion channel voltage sensors: structure, function, and pathophysiology. Neuron 67, 915-928 https://doi.org/10.1016/j.neuron.2010.08.021

Catterall WA. (2012): Voltage-gated sodium channels at 60: Structure, function and pathophysiology. J. Physiol. 590, 2577-2589 https://doi.org/10.1113/jphysiol.2011.224204

Catterall WA, Goldin AL, Waxman SG (2005): International Union of Pharmacology. XLVII Nomenclature and Structure-Function Relationships of Voltage-Gated Sodium Channels. Pharmacological Reviews 57, 397-409 https://doi.org/10.1124/pr.57.4.4

Catterall WA (2014): Structure and function of voltage-gated sodium channels at atomic resolution. Exp. Physiol. 99, 35-51 https://doi.org/10.1113/expphysiol.2013.071969

Charabarti N, Ing C, Payandeh J, Zheng N, Catterall WA, Pomès $\mathrm{R}$ (2013): Catalysis of $\mathrm{Na}+$ permeation in the bacterial sodium channel NavAb. PNAS 110, 11331-11336 https://doi.org/10.1073/pnas.1309452110

Chen R, Chung S. (2012): Binding modes of m-conotoxin to the bacterial sodium channel (NaVAb). Biophys. J. 102, 483-488 https://doi.org/10.1016/j.bpj.2011.12.041

Chomiak T, Hu B (2009): What is the optimal value of the g-ratio for myelinated fibers in the rat CNS.? A Theoretical approach. PLoS ONE 4, e7754 https://doi.org/10.1371/journal.pone.0007754

Chowdhury S, Chanda B (2012): Estimating the voltage-dependent free energy change of ion channels using the median voltage for activation. J. Gen. Physiol. 139, 3-17 https://doi.org/10.1085/jgp.201110722

Cole KS, Curtis HJ (1941): Membrane potential of the squid giant axon during current flow. J. Gen. Physiol. 24, 551-563 
https://doi.org/10.1085/jgp.24.4.551

Conway BE (1981): Ionic Hydration in Chemistry and Biophysics. Elsevier Scientific Pub. Co., Amsterdam

Debanne D, Campanac E, Bialowas A, Carlier E, Alcaraz G (2011): Axon physiology. Physiol. Rev. 91, 555-602 https://doi.org/10.1152/physrev.00048.2009

Debye P, Hückel E (1923): The theory of electrolytes. I. Lowering of freezing point and related phenomena. Physikalische Zeitschrift 24, 185-206

Deutsch S, Deutsch A (1993): Understanding the Nervous System: an Engineering Perspective. pp. 71, IEEE Press, New York https://doi.org/10.1109/9780470546864

Doyle DA, Cabral JM, Pfuetzner RA, Kuo A, Gulbis JM, Cohen SL, Chait BT, MacKinnon R (1998): The structure of the potassium channel: molecular basis of $\mathrm{K}+$ conduction and selectivity. Science 280, 69-77 https://doi.org/10.1126/science.280.5360.69

Fasman GD (1990): Prediction of Protein Structure and the Principles of Protein Conformation. pp. 273, Springer, New York

FitzHugh R (1961): Impulses and physiological states in theoretical models of nerve membrane. Biophys. J. 1, 445-466 https://doi.org/10.1016/S0006-3495(61)86902-6

FitzHugh R (1962): Computation of impulse initiation and saltatory conduction in a myelinated nerve fiber. Biophys. J. 2, 11-21 https://doi.org/10.1016/S0006-3495(62)86837-4

Friede RL, Beuche W (1985): A new approach toward analyzing peripheral nerve fiber populations. J. Neuropathol. Exp. Neurol. 44, 60-72 https://doi.org/10.1097/00005072-198501000-00005

Goldman DE (1943): Potential, impedance, and rectification in membranes. J. Gen. Physiol. 27, 37-60 https://doi.org/10.1085/jgp.27.1.37

Gramse G, Dols-Perez A, Edwards MA, Fumagalli L, Gomila G (2013): Nanoscale measurement of the dielectric constant of supported lipid bilayers in aqueous solutions with electrostatic force microscopy. Biophys. J. 104, 1257-1262 https://doi.org/10.1016/j.bpj.2013.02.011

Gutman GA, Chandy KG, Grissmer S, Lazdunski M, Mckinnon D, Pardo LA, Robertson GA, Rudy B, Sanguinetti MC, Stühmer W, Wang X (2005): International Union of Pharmacology. LIII Nomenclature and molecular relationships of voltage-gated potassium channels. Pharmacol. Rev. 57, 473-508 https://doi.org/10.1124/pr.57.4.10

Hall JE, Guyton AC (2011): The body fluid compartments: extracellular and intracellular fluids; interstitial fluid and edema. In: Textbook of Medical Physiology. pp. 264-278, Saunders Elsevier, Philadelphia

Hermann L (1879): Allgemeine nervenphysiologie. In: Handbuch der Physiologie. Vogel, Leipzig (in German)

Hindmarsh JL, Rose RM (1984): A model of neuronal bursting using three coupled first order differential equations. Proc. R. Soc. London, Ser. B. 221, 87-102 https://doi.org/10.1098/rspb.1984.0024

Hobbie RK, Roth BJ (2015): Intermediate Physics for Medicine and Biology. pp 169-170, Springer, New York https://doi.org/10.1007/978-3-319-12682-1

Hodgkin AL, Katz B (1949): The effect of sodium ions on the electrical activity of the giant axon of the squid. J. Physiol. 108, 37-77 https://doi.org/10.1113/jphysiol.1949.sp004310
Hodgkin AL, Huxley AF (1952a): Currents carried by sodium and potassium ions through the membrane of the giant axon of Loligo. J. Physiol. 116, 449-472

https://doi.org/10.1113/jphysiol.1952.sp004717

Hodgkin AL, Huxley AF (1952b): The components of membrane conductance in the giant axon of Loligo. J. Physiol. 116, 473-496 https://doi.org/10.1113/jphysiol.1952.sp004718

Hodgkin AL, Huxley AF (1952c): The dual effect of membrane potential on sodium conductance in the giant axon of Loligo. J. Physiol. 116, 497-506 https://doi.org/10.1113/jphysiol.1952.sp004719

Hodgkin AL, Huxley AF (1952d): A quantitative description of membrane current and its application to conduction and excitation in nerve. J. Physiol. 117, 500-544 https://doi.org/10.1113/jphysiol.1952.sp004764

Holcman JD, Yuste R (2015): The new nanophysiology: regulation of ionic flow in neuronal subcompartments. Nat. Rev. Neurosci. 11, 685-692 https://doi.org/10.1038/nrn4022

Horn R (2004): How S4 segments move charge. Let me count the ways. J.Gen. Physiol. 123, 1-4 https://doi.org/10.1085/jgp.200308975

Horn R (2005): How ion channels sense membrane potential. PNAS 102, 4929-4930 https://doi.org/10.1073/pnas.0501640102

Huxley AF, Stämpfli R (1949): Evidence for saltatory conduction in peripheral myelinated nerve fibers. J. Physiol. 108, 315-339 https://doi.org/10.1113/jphysiol.1949.sp004335

Islam MA (2004): Einstein-Smoluchowski Diffusion Equation: A Discussion. Physica Scripta 70, 2-3 https://doi.org/10.1088/0031-8949/70/2-3/008

Jack JJB, Noble D, Tsien RW (1975): Electric Current Flow in Excitable Cells. Clarendon Press, Oxford

Kier LB, Tombes RM (2013): Proton hopping: A proposed mechanism for myelinated axon nerve impulses. Chemistry \& Biodiversity 10, 596-599 https://doi.org/10.1002/cbdv.201200417

Kistler W, Gerstner W, Hemmen J (1997): Reduction of the Hodgkin-Huxley equations to a single-variable threshold model. Neural Comput. 9, 1015-1045

https://doi.org/10.1162/neco.1997.9.5.1015

Li QS, Wanderling S, Paduch M, Medovoy D, Singharoy A, McGreevy R, Villalba-Galea CA, Hulse RE, Roux B, Schulten K, Kossiakoff A, Perozo E (2014): Structural mechanism of voltage-dependent gating in an isolated voltage-sensing domain. Nat. Struct. Mol. Biol. 21, 244-252

https://doi.org/10.1038/nsmb.2768

Lide DR (2001): CRC Handbook of Chemistry and Physics. (82th ed.), CRC Press, Cleveland, Ohio

Lide DR (2005): CRC Handbook of Chemistry and Physics (86th ed.), CRC Press, Cleveland, Ohio

Loew LM (2012): Biophysical Journal 60 years after HodgkinHuxley. Biophys. J. 103, E1-E2 https://doi.org/10.1016/j.bpj.2012.06.002

Marcus YJ (1991): Thermodynamics of solvation of ions. Part 5.-Gibbs free energy of hydration at 298.15 K. J. Chem. Soc. Faraday Trans. 87, 2995-2999 https://doi.org/10.1039/FT9918702995 
Milo R, Philipps R (2015): Cell Biology by Numbers. pp. 89, Garland Science, New York

Moore JW (2015): Enhancing the Hodgkin-Huxley equations: simulations based on the first publication in the Biophysical Journal. Biophys. J. 109, 1317-1320 https://doi.org/10.1016/j.bpj.2015.08.008

Morris C, Lecar H (1981): Voltage oscillations in the barnacle giant muscle fiber. Biophys. J. 35, 193-213 https://doi.org/10.1016/S0006-3495(81)84782-0

Morris CE, Juranka PF (2007): Nav channel mechanosensitivity: activation and inactivation accelerate reversibly with stretch. Biophys. J. 93, 822-833 https://doi.org/10.1529/biophysj.106.101246

Nagumo J, Arimoto S, Yoshizawa S (1962): An active pulse transmission line simulating nerve axon. Proceeding of the IRE 50, 2061-2070 https://doi.org/10.1109/JRPROC.1962.288235

Neishabouri A, Faisal AA (2014): Saltatory conduction in unmyelinated axons: clustering of $\mathrm{Na}+$ channels on lipid rafts enables micro-saltatory conduction in C-fibers. Frontiers in Neuroanatomy 8, 109 https://doi.org/10.3389/fnana.2014.00109

Nunez PL, Srinivasan R (2006): Electric Fields of the Brain: The Neurophysics of EEG. Oxford University Press, New York https://doi.org/10.1093/acprof:oso/9780195050387.001.0001

Ogden D, Stanfield P (1994): Patch clamp techniques for single channel and whole-cell recording. Microelectrode techniques. In: The Plymouth Workshop Handbook. (Ed. D. Odgen), pp. 53-78, Cambridge, UK

Osorio N, Cathala L, Meisler MH, Crest M, Magistretti J, Delmas P (2010): Persistent Nav1.6 current at axon initial segments tunes spike timing of cerebellar granule cells. J. Physiol. 588, 651-669 https://doi.org/10.1113/jphysiol.2010.183798

Payandeh J, Scheuer T, Zheng N, Catterall WA (2011): The crystal structure of a voltage-gated sodium channel. Nature 475, 353-358 https://doi.org/10.1038/nature10238

Pods J, Schönke J, Bastian P (2013): Electrodiffusion models of neurons and extracellular space using the Poisson-Nernst-Planck equations - numerical simulation of the intra- and extracellular potential for an axon model. Biophys. J. 105, 242-254 https://doi.org/10.1016/j.bpj.2013.05.041

Purves D, Augustine GJ, Fitzpatrick D, Hall, WC, Lamantia AS, McNamara JO, Williams SM (2004): Neuroscience. (3rd ed.), Sunderland, Sinauer Associates, Inc, Massachusetts

Qi Z, Sokabe M (1999): Accelerated diffusion of $\mathrm{Na}+$ in a hydrophobic region revealed by molecular dynamics simulations of a synthetic ion channel. Biophys. Chem. 82, 183-193 https://doi.org/10.1016/S0301-4622(99)00118-0

Rall W (1962): Electrophysiology of dendritic neuron model. Biophys. J. 2, 145-167 https://doi.org/10.1016/S0006-3495(62)86953-7

Robbins CA, Tempel BL (2012): Kv1.1 and Kv1.2: Similar channels, different seizure models. Epilepsia 53, 134-141 https://doi.org/10.1111/j.1528-1167.2012.03484.x

Ruffner JA, Sperelakis N, Mann JE, Jr. (1980): Application of the Hodgkin-Huxley equations to an electric field model for interaction between excitable cells. J. Theor. Biol. 87, 129-152 https://doi.org/10.1016/0022-5193(80)90224-6
Rush AM, Dib-Hajj SD, Waxman SG (2005): Electrophysiological properties of two axonal sodium channels, Nav1.2 and Nav1.6, expressed in mouse spinal sensory neurons. J. Physiol. 564, 803-815 https://doi.org/10.1113/jphysiol.2005.083089

Rushton WAH (1951): A theory of the effects of fiber size in medullated nerve. J. Physiol. 115, 101-122 https://doi.org/10.1113/jphysiol.1951.sp004655

Sato C, Sato M, Iwasaki A, Doi T, Engel A (1998): The sodium channel has four domains surrounding a central pore. J. Struct. Biol. 121, 314-325 https://doi.org/10.1006/jsbi.1998.3990

Simeonova M, Gimsa J. (2006): The influence of the molecular structure of lipid membranes on the electric field distribution and energy absorption. Bioelectromagnetics 27, 652-666 https://doi.org/10.1002/bem.20259

Sheng M, Wyszynski M. (1992): Ion Channel targeting in neurons. Neuron 9, 271-284 https://doi.org/10.1016/0896-6273(92)90166-B

Shrager P (1988): Ionic channels and signal conduction in single remyelinating frog nerve fibers. J. Physiol. 404, 695-712 https://doi.org/10.1113/jphysiol.1988.sp017314

Shrager P (1987): The distribution of sodium and potassium channels in single demyelinated axons of the frog. J. Physiol. 392, 587-602 https://doi.org/10.1113/jphysiol.1987.sp016798

Shrager P (1989): Sodium channels in single demyelinated mammalian axons. Brain Res. 483, 149-154 https://doi.org/10.1016/0006-8993(89)90046-2

Sperelakis N (2002): An electric field mechanism for transmission of excitation between myocardial cells. Circ. Res. 91, 985-987 https://doi.org/10.1161/01.RES.0000045656.34731.6D

Szasz A, Szasz N, Szasz O (2011): Oncothermia: a new kind of oncologic hyperthermia. In: Oncothermia: Principles and Practices. pp. 126, Springer, Netherlands

Stühmer W, Ruppersberg JP, Schröter KH, Sakmann B, Stocker M, Giese KP, Perschke A, Baumann A, Pongs O (1989): Molecular basis of functional diversity of voltage-gated potassium channels in mammalian brain. EMBO J. 8, 3235-3244

Terlau H., Stühmer W. (1998): Structure and function of voltagegated ion channels. Naturwissenschaften 85, 437-444 https://doi.org/10.1007/s001140050527

Thomson W (1855): On the theory of the electric telegraph. Proc. R. Soc. 7, 382-399

Wang R, Tsuda I, Zhang Z (2015): A new work mechanism on neural activity. Int. J. Neural Syst. 25, 1450037 https://doi.org/10.1142/S0129065714500373

Waxman SG, Bennett MVL (1972): Relative conduction velocities of small myelinated and non-myelinated fibers in the central nervous system. Nature 238, 217-219

Wittmack EK, Rush AM, Hudmon A, Waxman SG, Dib-Hajj SD (2005): Voltage-gated sodium channel Nav1.6 is modulated by p38 mitogen-activated protein kinase. J. Neurosci. 28, 66216630

https://doi.org/10.1523/JNEUROSCI.0541-05.2005

Received: March 13, 2017

Final version accepted: April 21, 2017 